\title{
The Anorexia-Bulimia Spectrum: an Integrated Approach to Eating and Feeding Disorders
}

\author{
Mario Miniati', Simona Calugi ${ }^{2}$, Mario Savino e Mauro Mauri ${ }^{1}$ \\ ${ }^{1}$ Department of Clinical and Experimental Medicine University of Pisa, Italy \\ ${ }^{2}$ Department of Eating and Weight Disorders, Villa Garda Hospital
}

\section{Key words}

Anorexia Nervosa

Bulimia Nervosa

Binge-eating Disorder

Eating Spectrum

\begin{abstract}
Eating and Feeding Disorders are heterogeneous clinical conditions characterized by cognitive, interpersonal, and behavioural features. They might spread across a spectrum of severity, from mild or sub-threshold conditions, belonging to the realm of altered eating habits or body dysmorphic features, to severe disorders. The Anorexia-Bulimia Spectrum (ABS) aims at exploring and describing in a systematic manner this psychological/psychopathological area. The ABS model has been proposed to detect, in a lifetime perspective, signs and symptoms that might be considered as clinically relevant or not, but evenly associated with different levels of subjective impairment. To detect the eating disorder spectrum phenomenology, a structured clinical interview has been built and validated, the Anorexic-Bulimic Spectrum Clinical Interview (SCl-ABS). The paper describes its clinical significance and potential implications.
\end{abstract}

\section{Introduction}

The fifth edition of the Diagnostic and Statistical Manual of Mental Disorders, (DSM-5) (American Psychiatric Association, 2013) classifies a wide range of eating disorders (EDs) in the Feeding and Eating Disorders (FED) chapter, including not only the 'classic' ones, such as, anorexia nervosa (AN), bulimia nervosa (BN), and binge-eating disorder (BED), but also three disorders (avoidant/restrictive food intake disorder-ARFID, rumination disorder, and pica) previously included in a separate section dedicated to infancy/early childhood. In addition, the 'other specified feeding or eating disorders', and 'unspecified feeding/eating disorders' categories are still maintained, as dedicated to syndromes not presenting with typical symptoms or not fulfilling the threshold for the diagnosis.
It is not uncommon, in clinical practice, to evaluate patients who are not reaching the diagnostic threshold for DSM-5 Feeding and Eating disorders, but who may have a number of signs and symptoms interfering with dietary habits and body dissatisfaction levels, thus producing a relevant degree of subjective impairment (Mauri et al., 2002; Grange \& Loeb, 2007; Stice et al., 2009). In the past, only the most severe forms of eating disorders were considered in epidemiological and clinical studies (Rastam, 1992; Pope et al., 1984). More recently, an effort has been made to capture the great variability of eating disorders, with respect to both severity and clinical presentations (Grange \& Loeb, 2007). The model of the Anorexia-Bulimia Spectrum, as proposed by Mauri et al. $(2000,2002)$, encompasses a range of psychiatric phenomenology, dimensionally assessed, starting from the symptoms inclu-

Copyright @ 2019 Mario Miniati et al. This is an open-access article distributed under the terms of the Creative Commons Attribution License (CC $\mathrm{BY})$. The use, distribution or reproduction in other forums is permitted, provided the original author(s) and the copyright owner(s) are credited and that the original publication in this journal is cited, in accordance with accepted academic practice. No use, distribution or reproduction is permitted which does not comply with these terms.

Mario Miniati MD, PhD, (ه) mario.miniati@med.unipi.it

Ricevuto: 10 Giugno 2019; Accettato: 29 Giugno 2019; Pubblicato online: 8 Luglio 2019. doi:10.32044/ijedo.2019.07 
ded in the DSM descriptions. It refers both to typical and atypical signs and symptoms of the specific categories, as well as to sub-clinical signs and symptoms, temperamen$\mathrm{tal} /$ personality traits, behavioural patterns related to their 'core symptoms'. According to this model, eating spectrum symptoms may occur as prodromals to a fully developed disorder, as precursors of a not-yet-fully expressed disorder, as a mix of well-defined diagnostic categories, or as sequalae of a previously experienced full-fledged condition. The eating spectrum concept is intended to ascertain also whether symptoms and phenomena, that are usually considered 'soft' or 'clinically irrelevant', might, conversely, produce a subjective sufferance and an objective impairment (Cassano et al., 1997, 1999, 2002). In order to evaluate eating spectrum features using a standardized assessment, researchers of the Spectrum Project (1993-2019) developed and validated the self-report Anorexia-Bulimia Questionnaire (ABS-SR) designed to collect signs and symptoms of both $\mathrm{AN}$ and $\mathrm{BN}$, together with social aspects of EDs, which could affect the development and course of the disease or interfere with treatment response. The proposed spectrum approach overcomes the categorical dichotomy between $\mathrm{AN}$ and $\mathrm{BN}$ in a dimensional manner, assessing along a continuum, psychological and psychopathological characteristics of the two realms. Thus, in a trans-diagnostic perspective, these categories underscore key similarities, such as the over-evaluation of shape and weight, the preoccupation with eating, the dietary restraints and restrictions, the occurrence of binge eating and compensatory behaviors (Fairburn et al., 2003). We believe that a comprehensive approach, such as the one proposed by the anorexia-bulimia spectrum, might be useful to better define clinical and sub-clinical phenotypes among different subjects.

\section{The Structure of Anorexia-Bulimia Spectrum as declined in a specific instrument (ABS-SR)}

The Structured Clinical Interview for Anorexia-Bulimia Spectrum (SCI-ABS), also available in self-administration version, is an instrument composed of 134 items with dichotomous answer (yes/no) exploring nine psychopathological/psychological areas or 'domains', namely:

I. Attitudes and beliefs (items 1-7);

II. Weight History (items 8-12);

III. Self-esteem and satisfaction (items 13-23);
IV. Phobias (items 24-48);

V. Avoidant and compulsive behaviors (items 49-57);

VI. Weight maintenance (items 58-87);

VII. Eating dyscontrol (items 88-106);

VIII. Associated features and Physical consequences (items 107-126);

IX. Impairment and Insight (127-134).

The SCI-ABS represents an attempt to explore in a systematic manner both cognitive and behavioural aspects of EDs, in a lifetime perspective. The spectrum approach is more 'clinical' rather than 'theoretical', and overcomes the diagnostic dichotomy between $\mathrm{AN}$ and $\mathrm{BN}$, giving the instability of both categories, the frequent crossover between the two (Milos et al., 2005) and the sharing of most characteristics of the specific eating disorder psychopathology, irrespective of the DSM diagnosis (Fairburn et al., 2003). The first SCI-ABS four domains explore more in detail the cognitive aspects of eating spectrum; the domains from five to seven are mainly focused on behavioural aspects; the last two domains summarize some of the most relevant medical associated features that may produce functional impairment. Finally, the level of insight is explored by two questions on the ideal weight and on the subjective perception of weight as satisfactory or unsatisfactory. Acceptability and feasibility of the SCI-ABS, its psychometric properties, and the comparison of the SCI-ABS with two validated instruments (Eating Attitude Test, EAT, and Eating Disorder Inventory, EDI) have been already published by Mauri et al. $(2000,2002)$. Here we discuss and summarize the main contents of the SCI-ABS domains and the rationale for their conceptualization.

\section{Domain 'Attitudes and beliefs'}

The first domain includes the socio-cultural components and the subjective beliefs that may predispose to the development and the maintenance of anorexia-bulimia phenomenology, as already described in the available literature. Socio-epidemiological studies highlighted the role of habits, attitudes and beliefs related to the over-evaluation of the 'ideal physical form', especially in western countries, where thinness is emphasized as main indicator of health, beauty and social success (Franko \& Striegel-Moore, 2002). The differences in social attitudes and beliefs are especially relevant in adolescents. A considerable number of studies with representative samples of Afro-American and Caucasian adolescent girls reported more body dissatisfaction, dieting and disordered eating among Cauca- 
sian girls (Serdula et al., 1993; Striegel-Moore et al., 2000) than in Afro-American adolescents, enhancing the role of socio-cultural factors. Females of western countries have been found to report a thinner body ideal and be more likely to describe themselves as 'overweight' or 'too fat', with a high number of attempts to lose weight or dieting (Suisman et al., 2012). Thinness corresponds to 'competence', 'success', 'self-control', and 'sexual attraction'; conversely, obesity is often perceived as a symbol of laziness, self-indulgence and lack of will (Hausemblas et al., 2013). Taken as a whole, the studies on body dissatisfaction and disordered eating are raising questions both on predisposing and on protective psychosocial factors. Girls (such as the Afro-American adolescents) who seem to be protected from body dissatisfaction, might have powerful familial, peer and cultural influences enhancing and promoting the acceptance of larger body size. However, there is still a limited knowledge on the myriad of risk factors for eating spectrum disorders, including familial, peer, and cultural/ socioeconomic variables (Cook et al., 2014). The proposed spectrum model collects information in this area as part of the routine assessment and in close relationship with the other 'more clinical' or 'psychopathological' components of eating spectrum. This domain comprises seven questions exploring the above-mentioned 'general beliefs' about weight and physical appearance in a commonly utilized language, namely: 'Have you ever thought that being slim is one of the most important things to one's self- respect?' or '... a thin person is more sexually attractive and successful in his/her romantic life' or '... a thin person is more successful in his/her job?'; '..'eating slowly, just a little, or picking at one's food, is a sign of class or femininity?'; '...fat people look vulgar and disgusting?'; '...people should try to achieve the physical appearance of dancers, models or athletes?'; '...it is essential to keep up to date reading magazines about physical fitness, beauty, diets, and athletics?'.

\section{Domain 'Weight history'}

This psychological/psychopathological domain is included as part of the spectrum assessment in continuation with the previous one ('Attitudes and Beliefs'), considering that socio-cultural and familial aspects are often involved in determining behavioural response to an early history of overweight/obesity or underweight (Lebow et al., 2015). Family and personal history of subjects with eating spectrum might be characterized by childhood obesity, weight-related problems, early adoption of dietary regimes, or a history of underweight motivated by the refusal to a number of foods perceived as 'unhealthy' (Fairburn et al., 1998; Stein et al., 2006; Reba-Harrelson et al., 2010). Adolescents with overweight and obesity are targeted by public health campaigns, many of which are containing an inherent weight stigma, and they may be more likely to take drastic measures to control their weight (Hausemblas et al., 2013). Population-based samples of adolescents found that approximately $25 \%$ of female adolescents with obesity might use 'extreme' weight control behaviours, such as inducing vomiting, abusing laxatives, or using diet pills, a statistic that did not even account for 'milder' but still unhealthy weight control behaviours, such as fasting or smoking for weight control (Sunday et al., 1992). The tendency to the crumbling of food and the complaints about alleged digestive difficulties can be also predictors of the development of restrictive behaviours in childhood (Marchi et al., 1990). Weight history is explored in the ABS-SR with five questions on 'being overweight as child or adolescents', 'having a diet prescribed by the paediatrician', 'being underweight by adopting early restricting behaviours', 'being criticized by parents for the figure or weight', and finally 'being teased by friends and relatives for weight and body shape'.

\section{Domain 'Self-esteem and satisfaction'}

The persistence of low levels of self-esteem and satisfaction due to an excessive importance devoted to weight and body size is a diagnostic criterion both for DSM-5 AN and $\mathrm{BN}$ categories. Low self-esteem has been hypothesized to be a risk factor as well as a predisposing, precipitating, and maintaining mechanism of AN (Adamson, 2019), with a number of negative effects on intimate/interpersonal relationships (Miniati et al., 2018). Self-esteem maintains eating disorder psychopathology, especially contributing to concerns around shape, weight, and dietary restraint (Fairburn et al., 2003). However, this dimension seems to be a weak prognostic indicator for treatment response of EDs (Fairburn et al., 2003), considering that problems with low self-esteem may persist even after weight restoration and positive changes in eating behaviours (Button et al., 2002). Levels of self-esteem and satisfaction are explored in the SCI-ABS with eleven questions, encompassing both cognitive and behavioural aspects, such as 'thinking that weight and figure are central to self-esteem', that 'not being fat is the most important thing in life', through 'being happy, excited and full of energy while losing weight' or 'showing off when at a desired weight'. 


\section{Domain 'Phobias'}

This domain encompasses questions about worries regarding body shape/appearance and bodily functions/sensations. It is organized in four sub-headings.

\section{IV a. 'Body Dissatisfaction'}

Fear of weight gain and body image disturbances are core symptoms of EDs and diagnostic criteria for AN. In the most severe forms of AN Restricting Subtype (AN-R) some authors hypothesize that body image disturbance may reach a delusional level (Cassano et al., 2003; Miniati et al., 2016), thus suggesting the existence of an altered expression of dopaminergic genes as related to body image disturbances (Frieling et al., 2010). In line with the literature, the SCI-ABS body image disturbance is conceptualized as an altered 'body representation' with a selective attention to the areas with greater risk of fat accumulation, such as hips, thighs, buttocks, abdomen, or cheeks (Horne et al., 1991; Sunday et al., 1992; Fairburn et al., 2008). According to this approach, both the dissatisfaction with one's own body appearance, from the mildest to the most severe, and the manifestations associated with it (such as, the difficulty in finding satisfying clothing, the tendency to have more self-esteem when wearing clothes of a smaller size or the choice of large, shapeless, heavy dresses, preferably in dark colours because they are considered 'slimming' and suitable for covering the figure) are part of this domain. The SCI-ABS does not face the question of the co-occurrence of Body Dysmorphic Disorder (BDD) in ED patients. BDD is considered occurring in ED patients in $26-46 \%$ of cases, and the relationship between the two categories is still under debate (Jorgensen et al., 2001; Konstantakopoulos et al., 2012). Thus, BDD is characterized by an intense preoccupation with perceived defects in appearance that are unobservable or minor to others, but causing significant distress and having a negative impact on functioning, not differently from AN. However, by definition, areas of bodily concern in BDD are not better explained by concerns with body fat or weight in an individual who meet criteria for an EDs (APA, 2013). The two disorders remain differentiated to the areas of appearance concern and to the associated behaviours (Hartmann et al., 2013; Cerea et al., 2018).

\section{IV b. 'Weight gain phobia'}

The 'fear of gaining weight' is a nuclear characteristic of AN, with self-managed diets frequently characterizing its preliminary phases (Habermas et al., 1996). According to DSM-5, a fundamental difference between AN and Avoi-
dant/Restrictive Food Disorder (ARFID) is that restrictive eating in AN is driven by fear of weight gain, whether restrictive eating in ARFID is driven by sensitivity to the sensory characteristics of food, fear of aversive consequences of eating, and/or lack of interest in eating or food (APA, 2013). However, this difference is not completely clarified and despite most of patients with AN have an over-evaluation of shape and weight (and fear of gaining weight), recent studies highlighted that a limited number of patients with AN might have an over-evaluation of eating control per se, making unclear whether these patients are denying the underlying shape and weight concerns (Dalle Grave et al., 2008). The SCI-ABS explores this area with seven questions on extended periods of time characterized by the fear of becoming or remaining fat, namely: 'were you afraid of becoming fat, even when you were at or below your normal weight?', 'did you feel uncomfortable and guilty, even after eating small amounts of food such as chocolate, sweets, pasta or deep fried food, because you were afraid that you might gain weight?', 'did you buy a smaller size of clothes as an incentive to lose weight?', 'did you refuse to give up smoking for fear of gaining weight?', 'did you refuse to take a prescribed drug for fear of gaining weight?', 'did you consider essential have massages, take saunas, or use products to reduce cellulite?', 'did you consider essential to have regular bowel movements to avoid becoming fat or to keep a flat stomach?'. Authors decided to include also questions on the fear of gaining weight that can negatively interfere with adherence to pharmacological therapies due to the belief that some drugs, especially psychiatric ones, can cause an inevitable and irreversible weight gain.

\section{IV c. 'Secondary social phobia'}

Subjects with eating spectrum may avoid a number of social situations that pulled them in front of others. They can describe marked discomfort/avoidance of situations in which one is typically exposed to others' judgment, such as wearing new clothes, attending beaches or changing rooms and, in the most severe cases, undergoing medical examinations or having sexual intercourses.

\section{IV d. 'Fear of visceral perceptions'}

Patients with symptoms of the anorexia-bulimia spectrum often have an ideational polarization on visceral perceptions with poor tolerance to the sensations of swelling, fullness and constipation. A vague dyspeptic symptomatology is viewed as evidence that they have overeaten or are fat and favour body shape concerns and restrictive eating behaviours. Furthermore, the polarization on bowel 
function is frequent, motivated by the discomfort when the abdomen 'is not perceived as flat' and by the fear that a slowing down of peristalsis may 'favour' weight gain (Favaro et al., 2000). To counteract the sense of abdominal tension, patients frequently misuse anti-fermentative drugs, laxatives or diuretics that, however, contribute to maintain the eating disorder psychopathology, making increasingly more difficult to tolerate these feelings (Fairburn, 2008) and, in the long term, negatively affecting the peristaltic activity (Turner et al., 2000).

\section{Domain 'Avoidant and compulsive behaviours'}

This domain explores (again in an a-theoretical manner) the well-documented behaviours related to body image and eating concerns. In particular, the food checking behaviours that may be related to plan the whole day in accordance with what they intend to eat, with an increasing anxiety if the desired 'feeding program' is not completed or weighting the food or counting the calories (Fitzsimmons-Kraft et al., 2015). Moreover, the body image concerns behaviours, as body checking and body avoidance represent a direct expression of eating disorder psychopathology and contribute to its maintenance (Fairburn 2008). These aspects are explored with nine questions, from the need for 'regularly checking body dimensions with a tape measure' or the need for 'checking mirror everyday looking for fat', to the 'careful planning of the day according food intake'.

\section{Domain 'Weight maintenance'}

This domain is organized into two sub-domains, namely 'dietary habits' (20 items), and 'physical activity' (10 items) that are typical behavioural aspects of eating disorders. However, following the spectrum approach that considers relevant also more subtle manifestations that might be common in the general population (especially in female gender), questions encompass not only evident pathological behaviours (namely, 'taking medications or thyroid hormones to prevent weight gain', 'taking amphetamines to decrease appetite and lose weight', 'self-induced vomiting', 'use/ abuse of laxatives and diuretics'), but also more 'accepted and shared' habits, such as 'using stairs, walk or using bike to spend energy', 'fasting for a day when eaten too much the day before', or 'to increase physical activity after eating a lot to control weight', that might be all considered part of a 'healthy approach' to food and weight control. Recently, the widespread of these behaviours in the general population and the promotion of lifestyles characterized by a 'rigid' thinking regarding nutrition and the pursuing of this goal through diets, the focus on food preparation, and a ritualized pattern of eating, has prompted a number of studies on exploring the boundaries between eating disorders, healthy habits and orthorexia (Aksoydan \& Camci, 2009). However, 'orthorexic individuals' are typically concerned by the 'quality', as opposed to the 'quantity' of food in one's diet and could represent a variant of ARFID (Donini et al., 2004).

\section{Domain 'Eating Dyscontrol'}

This domain describes a typical core symptomatology of the overall spectrum of eating disorders, except for the 'pure' AN-R, whose patients are characterized by a rigid and persistent control over food intake. Nineteen questions explore both behavioural aspects (such as eating a large amount of food in a short period or eating a large amount of food when not hungry), and feelings (such as, being embarrassed when eating, being disgusted or depressed or very guilty right after overeating).

\section{Domain 'Associated Features and Consequences'}

This domain is organized in three sub-domains, namely 'Impulse control', 'Personality', and 'Physical Consequences'. The first sub-domain (Impulse control) is in line with the previous domain (Eating Dyscontrol) but refers to a wider form of difficulties in maintaining control over a number of different impulses, such as substances abuse (from caffeine, tobacco and alcohol to opiates, cocaine, marijuana and ecstasy), the impulse of stealing something, or the presence of self-injuring. These aspects have been selected following the wide body of literature on the comorbidity between bulimic spectrum disorders and borderline personality traits (Mitchell et al., 1992; Loxton \& Dawe, 2001; Askenazy et al., 2003; Bulik et al., 2004). The following sub-domain (Personality) explores more in detail personality traits belonging to opposite sites of the spectrum, namely to obsessive-compulsive personality traits and borderline personality traits, with eleven questions (e.g., 'having the need of feeling sexually attractive in order to feel worthwhile', 'having feelings of inadequacy in relationships', 'being perfectionist, intolerant to other people'). The five questions on physical consequences of eating spectrum signs and symptoms of the third sub-domain (Physical Consequences) are less related to the two previous sub-do- 
mains and are typical of the most severe or enduring forms of AN and BN. These questions about being disinterested in sex, particularly sensitive to cold or having a yellowish coloration of skin are introducing the last domain on the level of impairment and insight.

\section{Domain 'Impairment and Insight'}

The level of insight and the interference that the symptoms of the anorexia-bulimia spectrum might have in the patient's daily life can vary along a continuum of severity. Evidence from epidemiological and clinical studies shows that, even if the specific criteria for the categorical diagnosis of eating disorders are not met, sub-threshold symptoms can cause significant distress and interfere with individual functioning levels. Insight correlates negatively with the severity of body image disturbance (Cassano et al., 2003; Miniati et al., 2016).

\section{Discussion}

The majority of studies in eating disorders (ED) is focused on AN, BN and BED. The atypical, incomplete, residual or sub-threshold forms of EDs have been included in a wastebasket category of DSM-IV-TR, namely, the eating disorder not otherwise specified (EDNOS). The DSM-5 reconfigured EDNOS as 'other specified feeding or eating disorder' (OSFED). OSFED encompasses a number of heterogeneous syndromes, such as 'atypical anorexia nervosa' (atypical-AN), 'purging disorder' (PD), 'sub-threshold bulimia nervosa' (Sub-BN), 'sub-threshold binge eating disorder' (Sub-BED), and 'night eating syndrome' (NES). This EDNOS reshaping aimed at enhancing the study of those eating syndromes that did not reach the threshold for a diagnostic categorization into the 'classic forms' of eating disorders but that were clinically relevant. This was an attempt to better define clinical phenotypes of eating and feeding disorders, still using a categorical approach. Conversely, the proposed 'eating spectrum model' by Mauri et al. $(2000,2002)$ refers to an integrated view, with the assessment of a number of psychopathological/psychological dimensions (domains) within the DSM diagnostic categories. This approach has prompted research mainly in other two fields than eating disorders, namely panic-agoraphobic and mood disorders spectra (Cassano et al., 1997; Cassano et al., 1999; the spectrum instruments are all available for download at the Spectrum Website: http://www.spectrumproject.org/intro.html). For example, data emerging from the studies with the mood spectrum approach suggested the existence of a continuum from 'pure mania' to 'pure depression', without a clear cut-off between the two realms, confirming the need for a probabilistic approach towards mood disorders (Angst \& Cassano, 2005; Phelps et al., 2008; Mitchell et al., 2008). In a similar manner, literature on eating disorders is suggesting a continuum between $\mathrm{AN}$ and $\mathrm{BN}$, as demonstrated by the studies on the diagnostic instability of eating disorders across the lifespan (Milos et al., 2005) and as confirmed by the trans-diagnostic theory and treatment of eating disorders (Fairburn et al., 2003; Fairburn 2008). Starting from this point of observation, the anorexia-bulimia spectrum tried to describe in a systematic and unitary manner, with a validate instrument, the wide halo of phenomenology surrounding the 'core' features of each DSM eating/feeding category overcoming the classic AN/BN dichotomy. The potential implications and the conceptual and methodological advantages of the proposed spectrum model have been again described mainly for the mood spectrum assessment (Fagiolini et al., 1999). Finding in this field have been recently summarized in a review on mood spectrum model, reconsidered in the light of DSM-5 (Benvenuti et al., 2015). The anorexia-bulimia spectrum has been constructed with the same rationale, and with the aim to identify and describe the sub-threshold conditions, exploring the lifetime experience of an individual. The SCI-ABS and the ABS-SR have been validated to highlight both patient vulnerabilities for the development of a manifest ED and the sequalae of a previous disorder that may require clinical attention and be monitored over time. These instruments, if used in the clinical practice, could be a good guide in psychological and psychiatric assessment and could furnish relevant cues to investigate predictors of treatment. More in detail, we believe that the usefulness of these instrument is twofold: for clinical purposes, spectrum instruments are able to detect and to systematically assess not only the 'core' and typical symptoms of eating disorders, but also their more subtle or atypical manifestations; for research purposes, the use of anorexia-bulimia spectrum domains should be helpful for the selection of homogeneous subgroups of patients for brain-imaging studies and for the exploration of pathophysiology of eating disorders at molecular level. Moreover, when used in combination with more traditional categorical assessment approaches (such as the SCID), the Anorexia-Bulimia Spectrum assessment may represent a sensitive tool for the evaluation of those eating features that may modify the effectiveness of treatments. Current results with the spectrum questionnaires and interviews 
suggest that tailored psychotherapeutic and pharmacologic strategies may be necessary to treat in the optimal manner patients with the same Axis I diagnosis, but different spectrum profiles.

\section{References}

Adamson, J., Ozenc, C., Baillie, C., \& Tchanturia, K. (2019). Self-Esteem Group: Useful Intervention for Inpatients with Anorexia Nervosa? Brain Sciences, 9(1), 12.

Aksoydan, E., \& Camci, N. (2009). Prevalence of orthorexia nervosa among Turkish performance artists. Eating and Weight Disorders-Studies on Anorexia, Bulimia and Obesity, 14(1), 33-37.

Angst, J., \& Cassano, G. (2005). The mood spectrum: improving the diagnosis of bipolar disorder. Bipolar Disorders, 7, 4-12.

Askénazy, F. L., Sorci, K., Benoit, M., Lestideau, K., Myquel, M., \& Lecrubier, Y. (2003). Anxiety and impulsivity levels identify relevant subtypes in adolescents with at-risk behavior. Journal of Affective Disorders, 74(3), 219-227.

Association, A. P. (2013). Diagnostic and statistical manual of mental disorders (DSM-5). American Psychiatric Pub.

Benvenuti, A., Miniati, M., Callari, A., Mariani, M. G., Mauri, M., \& Dell'Osso, L. (2015). Mood Spectrum Model: Evidence reconsidered in the light of DSM-5. World Journal of Psychiatry, 5(1), 126.

Bulik, C. M., Klump, K. L., Thornton, L., Kaplan, A. S., Devlin, B., Fichter, M. M., Crow, S. (2004). Alcohol use disorder comorbidity in eating disorders: a multicenter study. The Journal of Clinical Psychiatry.

Button, E. J., \& Warren, R. L. (2002). Self-image in anorexia nervosa 7.5 years after initial presentation to a specialized eating disorders service. European Eating Disorders Review: The Professional Journal of the Eating Disorders Association, 10 (6), 399-412.

Cassano, G. B., Dell'Osso, L., Frank, E., Miniati, M., Fagiolini, A., Shear, K., Maser, J. (1999). The bipolar spectrum: a clinical reality in search of diagnostic criteria and an assessment methodology. Journal of Affective Disorders, 54(3), 319-328.

Cassano, G. B., Frank, E., Miniati, M., Rucci, P., Fagiolini, A., Pini, S., Maser, J. D. (2002). Conceptual underpinnings and empirical support for the mood spectrum. Psychiatric Clinics, 25(4), 699-712.

Cassano, G. B., Michelini, S., Shear, M. K., \& Coli, E. (1997). The panic-agoraphobic spectrum: a descriptive approach to the assessment and treatment of subtle symptoms. The American Journal of Psychiatry, 154(6), 26.

Cassano, G. B., Miniati, M., Pini, S., Rotondo, A., Banti, S., Borri, C., Mauri, M. (2003). Six-month open trial of haloperidol as an adjunctive treatment for anorexia nervosa: A preliminary report. International Journal of Eating Disorders, 33(2), 172-177.

Cerea, S., Bottesi, G., Grisham, J. R., \& Ghisi, M. (2018). Non-weight-related body image concerns and Body Dysmorphic Disorder prevalence in patients with Anorexia Nervosa. Psychiatry Research, 267, 120-125.

Cook, B., Engel, S., Crosby, R., Hausenblas, H., Wonderlich, S., \& Mitchell, J. (2014). Pathological motivations for exercise and eating disorder specific health-related quality of life. International Journal of Eating Disorders, 47(3), 268-272.

Dalle Grave, R., Calugi, S., \& Marchesini, G. (2008). Underweight eating disorder without over-evaluation of shape and weight: Atypical anorexia nervosa? International Journal of Eating Disorders, 41(8), 705-712.

Donini, L. M., Marsili, D., Graziani, M. P., Imbriale, M., \& Cannella, C. (2004). Orthorexia nervosa: a preliminary study with a proposal for diagnosis and an attempt to measure the dimension of the phenomenon. Eating and Weight Disorders-Studies on Anorexia, Bulimia and Obesity, 9(2), 151-157.

Fagiolini, A., Dell'Osso, L., Pini, S., Armani, A., Bouanani, S., Rucci, P., Katherine Shear, M. (1999). Validity and reliability of a new instrument for assessing mood symptomatology: the Structured Clinical Interview for Mood Spectrum (SCI-MOODS). International Journal of Methods in Psychiatric Research, 8(2), 71-82.

Fairburn, C. G. (2008). Cognitive behavior therapy and eating disorders. Guilford Press.

Fairburn, C. G., Cooper, Z., \& Shafran, R. (2003). Cognitive behaviour therapy for eating disorders: A "transdiagnostic" theory and treatment. Behaviour Research and Therapy, 41(5), 509-528.

Fairburn, C. G., Doll, H. A., Welch, S. L., Hay, P. J., Davies, B. A., \& O'Connor, M. E. (1998). Risk factors for binge eating disorder: a community-based, case-control study. Archives of General Psychiatry, 55(5), 425-432.

Favaro, A., Caregaro, L., Burlina, A. B., \& Santonastaso, P. (2000). Tryptophan levels, excessive exercise, and nutritional status in anorexia nervosa. Psychosomatic Medicine, 62(4), 535-538.

Fitzsimmons-Craft, E. E., Accurso, E. C., Ciao, A. C., Crosby, R. D., Cao, L., Pisetsky, E. M., Engel, S. G. (2015). Restrictive eating in anorexia nervosa: 
Examining maintenance and consequences in the natural environment. International Journal of Eating Disorders, 48(7), 923-931.

Franko, D. L., \& Striegel-Moore, R. H. (2002). The role of body dissatisfaction as a risk factor for depression in adolescent girls: Are the differences Black and White? Journal of Psychosomatic Research, 53(5), 975-983.

Frieling, H., Römer, K. D., Scholz, S., Mittelbach, F., Wilhelm, J., De Zwaan, M., Bleich, S. (2010). Epigenetic dysregulation of dopaminergic genes in eating disorders. International Journal of Eating Disorders, 43(7), 577-583.

Grange, D. le, \& Loeb, K. L. (2007). Early identification and treatment of eating disorders: prodrome to syndrome. Early Intervention in Psychiatry, 1(1), 27-39.

Habermas, T. (1996). In defense of weight phobia as the central organizing motive in anorexia nervosa: Historical and cultural arguments for a culturesensitive psychological conception. International Journal of Eating Disorders, 19(4), 317-334.

Hartmann, A. S., Greenberg, J. L., \& Wilhelm, S. (2013). The relationship between anorexia nervosa and body dysmorphic disorder. Clinical Psychology Review, 33(5), 675-685.

Hausenblas, H. A., Campbell, A., Menzel, J. E., Doughty, J., Levine, M., \& Thompson, J. K. (2013). Media effects of experimental presentation of the ideal physique on eating disorder symptoms: A meta-analysis of laboratory studies. Clinical Psychology Review, 33(1), 168-181.

Horne, R. L., Van Vactor, J. C., \& Emerson, S. (1991). Disturbed body image in patients with eating disorders. The American Journal of Psychiatry, 148(2), 211.

Jorgensen, L., Castle, D., Roberts, C., \& Groth-Marnat, G. (2001). A clinical validation of the Dysmorphic Concern Questionnaire. Australian \& New Zealand Journal of Psychiatry, 35(1), 124-128.

Konstantakopoulos, G., Varsou, E., Dikeos, D., Ioannidi, N., Gonidakis, F., Papadimitriou, G., \& Oulis, P. (2012). Delusionality of body image beliefs in eating disorders. Psychiatry Research, 200(2-3), 482-488.

Lebow, J., Sim, L. A., \& Kransdorf, L. N. (2015). Prevalence of a history of overweight and obesity in adolescents with restrictive eating disorders. Journal of Adolescent Health, 56(1), 19-24.

Loxton, N. J., \& Dawe, S. (2001). Alcohol abuse and dysfunctional eating in adolescent girls: The influence of individual differences in sensitivity to reward and punishment. International Journal of Eating Disorders, $29(4), 455-462$.
Marchi, M., \& Cohen, P. (1990). Early childhood eating behaviors and adolescent eating disorders. Journal of the American Academy of Child \& Adolescent Psychiatry, 29(1), 112-117.

Mauri, M., Borri, C., Baldassari, S., Benvenuti, A., Cassano, G., Rucci, P., Shear, M., Grochocinski, V., Frank, E. and Maser, J. (2000). Acceptability and psychometric properties of the Structured Clinical interview for Anorexic-Bulimic Spectrum (SCI-ABS). International Journal of Methods in Psychiatric Research, 9(2), 68-78.

Mauri, M., Borri, C., Banti, S., Baldassari, S., Rucci, P., Cassano, G. B. (2002). The anorexic-bulimic spectrum in patients with eating disorders, mood disorders and controls. Journal of Psychopathology, 8(2).

Milos, G., Spindler, A., Schnyder, U., \& Fairburn, C. G. (2005). Instability of eating disorder diagnoses: prospective study. The British Journal of Psychiatry, 187(6), 573-578.

Miniati, M., Callari, A., Maglio, A., \& Calugi, S. (2018). Interpersonal psychotherapy for eating disorders: current perspectives. Psychology Research and Behavior Management, 11, 353.

Miniati, M., \& Marazziti, D. (2017). Psychopharmacological options for adult patients with anorexia nervosa: the patients' and carers' perspectives integrated by the spectrum model. CNS Spectrums, 1-2.

Miniati, M., Mauri, M., Ciberti, A., Mariani, M. G., Marazziti, D., \& Dell'Osso, L. (2016). Psychopharmacological options for adult patients with anorexia nervosa. CNS Spectrums, 21(2), 134-142.

Mitchell, J. E., Fletcher, L., Gibeau, L., Pyle, R. L., \& Eckert, E. (1992). Shoplifting in bulimia nervosa. Comprehensive Psychiatry, 33(5), 342-345.

Mitchell, P. B., Goodwin, G. M., Johnson, G. F., \& Hirschfeld, R. M. (2008). Diagnostic guidelines for bipolar depression: a probabilistic approach. Bipolar Disorders, 10(1p2), 144-152.

Phelps, J., Angst, J., Katzow, J., \& Sadler, J. (2008). Validity and utility of bipolar spectrum models. Bipolar Disorders, 10(1p2), 179-193.

Pope, H. G., Hudson, J. I., \& Yurgelun-Todd, D. (1984). Anorexia nervosa and bulimia among 300 suburban women shoppers. The American Journal of Psychiatry.

Rastam, M. (1992). Anorexia nervosa in 51 Swedish adolescents: premorbid problems and comorbidity. Journal of the American Academy of Child \& Adolescent Psychiatry, 31(5), 819-829.

Reba-Harrelson, L., Von Holle, A., Hamer, R. M., Torgersen, L., Reichborn-Kjennerud, T., \& Bulik, C. M. 
(2010). Patterns of maternal feeding and child eating associated with eating disorders in the Norwegian Mother and Child Cohort Study (MoBa). Eating Behaviors, 11(1), 54-61.

Serdula, M. K., Collins, M. E., Williamson, D. F., Anda, R. F., Pamuk, E., \& Byers, T. E. (1993). Weight control practices of US adolescents and adults. Annals of Internal Medicine, 119(7_Part_2), 667-671.

Stein, A., Woolley, H., Cooper, S., Winterbottom, J., Fairburn, C. G., \& Cortina-Borja, M. (2006). Eating habits and attitudes among 10-year-old children of mothers with eating disorders: longitudinal study. The British Journal of Psychiatry, 189(4), 324-329.

Stice, E., Marti, C. N., Shaw, H., \& Jaconis, M. (2009). An 8-year longitudinal study of the natural history of threshold, subthreshold, and partial eating disorders from a community sample of adolescents. Journal of Abnormal Psychology, 118(3), 587.

Striegel-Moore, R. H., Schreiber, G. B., Lo, A., Crawford, P., Obarzanek, E., \& Rodin, J. (2000). Eating disorder symptoms in a cohort of 11 to 16-year-old black and white girls: The NHLBI growth and health study. International Journal of Eating Disorders, 27(1), 49-66.
Suisman, J. L., O'connor, S. M., Sperry, S., Thompson, J. K., Keel, P. K., Burt, S. A., Klump, K. L. (2012). Genetic and environmental influences on thinideal internalization. International Journal of Eating Disorders, 45(8), 942-948.

Sunday, S. R., Einhorn, A., \& Halmi, K. A. (1992). Relationship of perceived macronutrient and caloric content to affective cognitions about food in eatingdisordered, restrained, and unrestrained subjects. The American Journal of Clinical Nutrition, 55(2), 362-371.

Turner, J., Batik, M., Palmer, L. J., Forbes, D., \& McDermott, B. M. (2000). Detection and importance of laxative use in adolescents with anorexia nervosa. Journal of the American Academy of Child \& Adolescent Psychiatry, 39(3), 378-385. 